\title{
Benign Tumors of Bone
}

\section{Subbarao K}

Padmshri Prof. Dr. Kakarla Subbara, Hyderabad, India.

Benign tumors of bone are common while malignant tumors are rare. Benign tumors constitute about $75 \%$ of all bone tumors excluding metastasis. Most of these tumors do not need surgery as diagnosis is made on plain films. $2 \%$ of all benign tumors may undergo malignancy. Several of these bone tumors present clinically with pain, swelling and symptoms of compression of vascular and neural structures. Many of them present with history of trauma and a pathological fracture. Some of them are called as "leave me alone" lesions as biopsy or surgery is unnecessary. The following table I gives the classification according to the tissue of origin

Table I: Classification of benign tumors of bone according to the tissue of origin.

\begin{tabular}{|l|}
\hline - Osseous tissue \\
\hline - Cartilaginous tissue \\
\hline - Fibrous tissue \\
\hline - Mascular tissue \\
\hline
\end{tabular}

Radiological investigations include the following table II:

Table II: Radiological investigations

- Conventional

- Ultrasonography

- MDCT

- Pet CT

- Radionuclide scanning

- MRI

All these investigations may not be necessary in any given patient. The general principles applied in the diagnosis of bone tumors are given in table III

Table III: General principles applied in the diagnosis of bone tumors.

- Age and Sex

- Single or Multiple Lesions

- Type of Bone involved

- Site of the lesion within the bone

- Site of origin of the lesion

- Nature and extent of bone change

- Appearance of the transitional zone

- Nature of the tumour matrix

- Type of periosteal reaction

- Soft tissue involvement

The radiological findings in a benign tumor include narrow transitional zone, well defined border, intact periosteum and minimal soft tissue swelling:

Tumors of osseous tissue include osteoma, osteoid osteoma, osteoblastoma etc., osteomas constitute condensed bone with well defined border. They are more common in para nasal sinuses, skull and long bones (Fig.1ab). Most of them are detected incidentally. They should not be mistaken for bone island, however large they may be (Fig. 2ab). Osteomas of the frontal sinus may erode the bone and produce pneumoencephalocele. Potential of malignancy does not exist (Fig.2c). Multiple osteomata with intestinal polyposis goes with the name of Gardner syndrome (Fig. 3). 


\section{OSTEOID OSTEOMA}

Osteoid osteoma occurs in first four decades of life and is more common in men. $2 / 3^{\text {rd }}$ effect appendicular skeleton specially proximal and distal segments of femur and proximal half of tibia. Computed Tomography (CT), isotopic scanning and MRI may be necessary to confirm the diagnosis and to demonstrate the nidus (Fig. 4abc). It is less common in axial skeleton. Skull and facial bones are affected very rarely. Morphologically and anatomically there are four types table IV:

Table IV: Morphological and anatomical location of Osteoid osteoma:

- Intra Cortical

- Periosteal

- Spongiosal

- Subarticular

The essential point in the radiological diagnosis is the identification of central calcifications surrounded by ovoid translucency. The average diameter of the nidus is between $5 \mathrm{~mm}$ to $1.5 \mathrm{~cm}$ (Fig. 5). If the nidus is more than $2 \mathrm{~cm}$ it belongs to the category of osteoblastoma. Intracortical osteoid osteoma produces dense sclerosis around the nidus. Subperiosteal type produces periosteal reaction while spongiosal type produces very little reactive bone (Fig. 6). Subarticular osteoid osteoma simulates arthritis as it produces synovial reactions (Fig. 7). In the vertebra it occurs in the neural arch and produces scoliosis due to pain. CT scanning is essential to identify the nidus (Fig. $8)$.

It is of significance that CT and MRI play a better role than three phase technetium $99 \mathrm{~mm}$ isotopic scanning (Fig. 9ab and 10)

\section{OSTEOBLASTOMA}

Osteoblastoma is encountered in the first four decades of life but common in $2^{\text {nd }}$ and $3^{\text {rd }}$ decades. The sites of predilection include neural arches of spine as well as the vertebral bodies. Diaphysis of long bones is also a common site. The other sites are less common.

Radiological features include a large nidus more than $2 \mathrm{~cm}$ in diameter. New bone formation occurs with periosteal reaction specially in long bones. MDCT plays a major role in identifying osseous matrix (Fig. $11 \& 12$ ). Aneurysmal bone cyst may superimpose and may be associated with osteoblastoma. In long bones, periosteal reaction may be prominent (Fig. 13).

\section{CARTILAGINOUS TUMORS}

Cartilaginous tumors constitutes major of all the benign tumors. The classification of these tumours is included in the table V:

\section{Table V: Classification of Cartilaginous Tumors.}

\begin{tabular}{|l|l|}
\hline \multicolumn{1}{|c|}{ MONOSTOTIC } \\
\hline - & Enchondroma \\
\hline - & Osteochondroma \\
\hline - & Puxta Cortical chondroma \\
\hline - & Chondroal chondroma \\
\hline - & Chondroblastoma \\
\hline - & Chondromyxoid Fibroma \\
\hline - & Post traumatic \\
\hline
\end{tabular}

\begin{tabular}{|c|c|}
\hline POL & YOSTOTIC \\
\hline$\bullet$ & Multiple enchondromata \\
\hline$\bullet$ & Metachondromatosis (Hereditary) \\
\hline • & Ollier's dyschondrodysplasia \\
\hline & Maffuci’s Syndrome \\
\hline 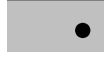 & Multiple osteochondromata \\
\hline
\end{tabular}

Diagnosis of chondroid tumours mainly rests on conventional radiology and is made by the anatomical location, transitional zone and mineralization of matrix. The calcified matrix may be minimal or heavy. When there is no mineralization of cortex, it is difficult to make a firm diagnosis. In these instances CT or MRI may be helpful. Endosteal scalloping, thick periosteal 


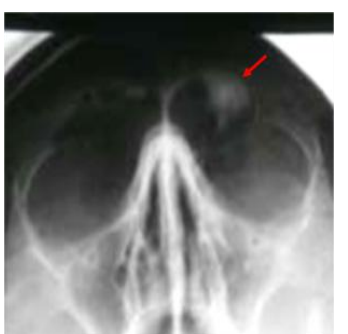

Fig.1a: Osteoma in left frontal sinus

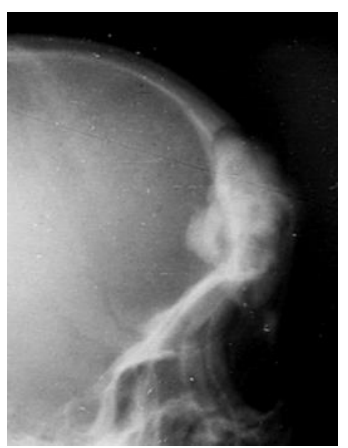

Fig.1b: Lateral view of skull showing large osteoma of frontal sinus.

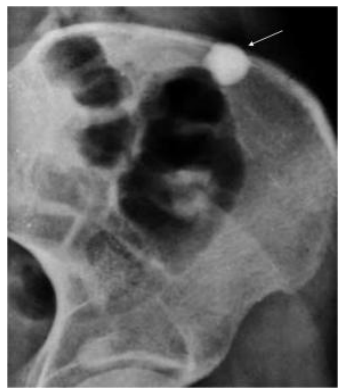

Fig.2a: Bone Island (Insular compacta)

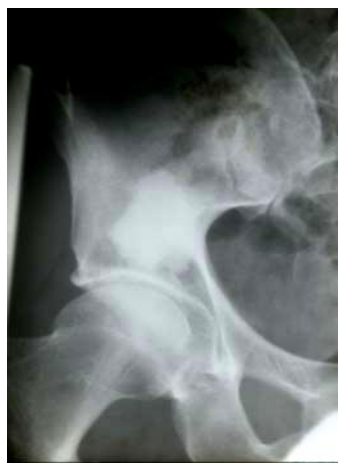

Fig.2b: Giant bone island in iliac bone

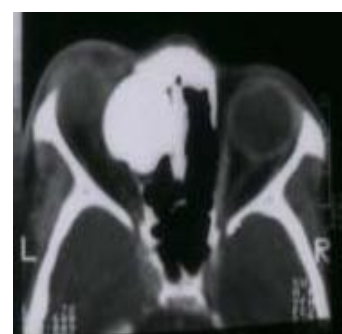

Fig.2c: CT Ethmoid osteoma with pressure on the eye ball.

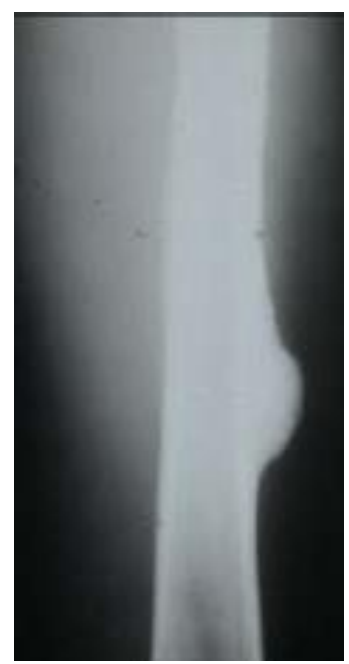

Fig.3: Flat osteoma of femoral shaft in

Gardner syndrome with polyposis coli.

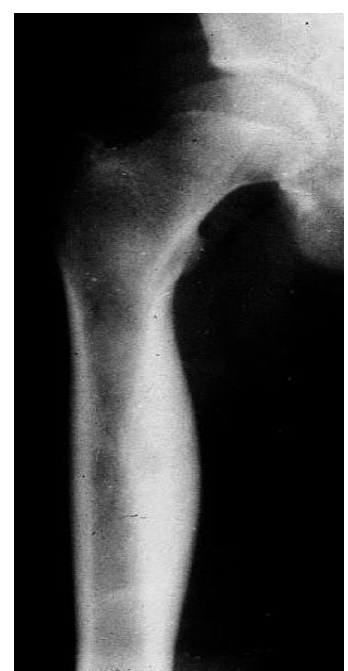

Fig.4a: Intracortical osteoid osteoma. Dense reactive bone obscures the nidus.

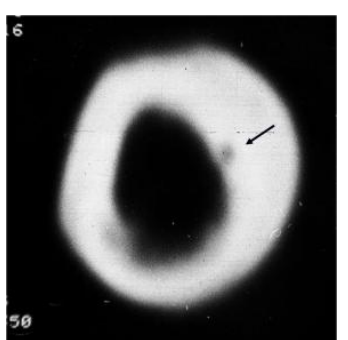

Fig. 4b: CT of femur of same patient showing the nidus.

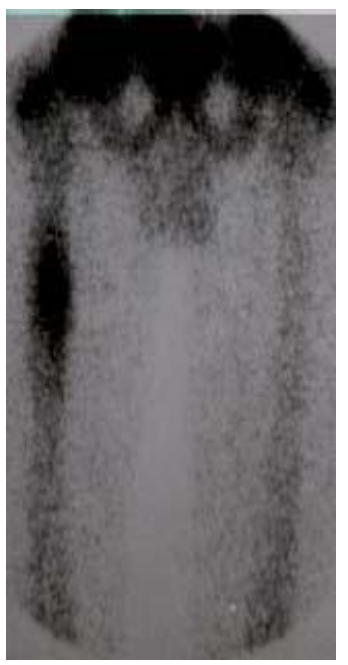

Fig.4c: Technetium 99mm scanning showing hot spot at the site of osteoid osteoma.

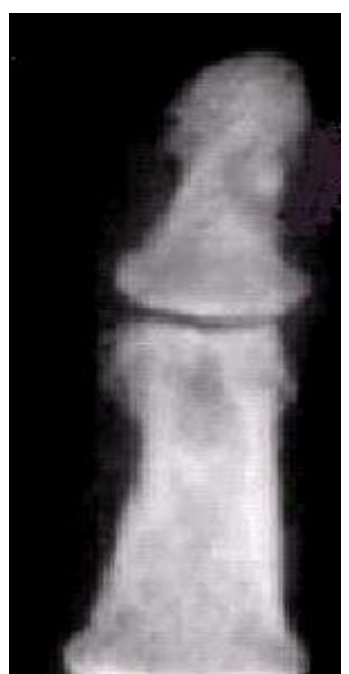

Fig.5: Osteoid osteoma in the distal phalanx of the thumb with central calcification.

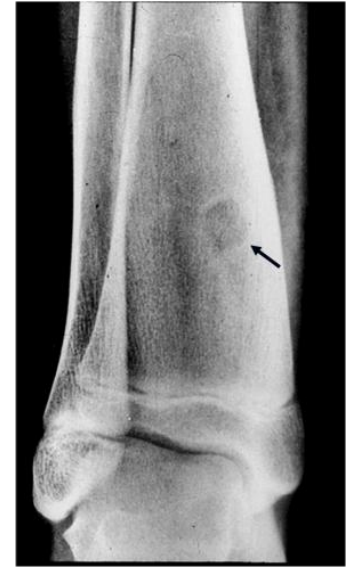

Fig. 6: Spongiosal type of osteoid osteoma in tibia producing minimal reactive bone

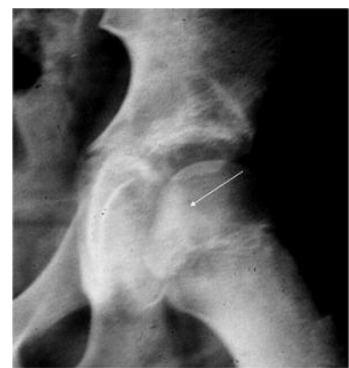

Fig.7 : Subarticular osteoid osteoma (arrow) with widening of the joint space due to synovitis.

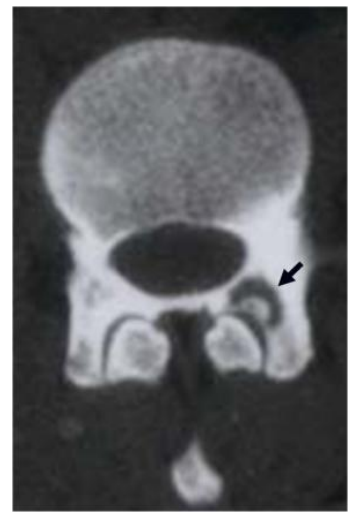

Fig.8: CT of spine showing osteoid osteoma(target sign) 
reaction and cortical hook when present may add to the correct diagnosis.

\section{ENCHONDROMA}

It is generally asymptomatic except when there is a trauma where a pathological fracture is present (Fig.14). The age is between $10-30$ yrs. When multiple these are located in tubular bones of hands (Fig.15). Multiple enchondromata in the hands is also called Ollier's syndrome. Other sites include long bones including ribs (Fig. 16abc). Enchondroma is located during the central medullar canal in the metaphysis. The incidence is $3-17 \%$ of biopsied primary bone tumors and it is second most common cartilage containing tumor. Etiology is continued growth of residual benign rests of cartilage displaced from the growth plate. The calcifications of the matrix may assume the shape of punctuate, arc like, circular, nodular, or a mixture of these (Fig. 17ab). Another finding in cartilaginous tumors is the presence of a hook at the margins which has not been described in the literature (Fig. 18).

Periosteal chondroma is also called juxta cortical chondroma and is diagnosed by the anatomical location and the presence of calcific matrix (Fig. 19ab).

The complications of enchondroma include pathological fracture and malignant degeneration particularly in long-bone enchondromas and the incidence is between 15 and $20 \%$. In the differential diagnosis, epidermoid inclusion cyst (phalangeal tuft), with history of trauma, unicameral bone cyst (rare in hands, more radiolucent), giant cell tumor of tendon sheath (commonly erodes bone, soft-tissue mass outside bone) fibrous dysplasia (mostly polyostotic) and bone infarct (Fig. 20ab).

Maffucci syndrome constitutes multiple enchondromata and soft tissue hemangiomas. It is actually a non hereditary mesodermal dysplasia. There is increased prevalence of malignancies in the bone, ovary, duodenum and central nervous system. The diagnosis of Maffucci syndrome is made by the presence of enchondromata and presence of phleboliths in the soft tissue hemangiomas (Fig. 21).

Soft tissue chondromas are also encountered without the involvement of underlying bones. They show amorphous, lumpy and homogenous calcifications (Fig.22ab). They may be seen in intra and extra articular locations (Fig. 23ab).

Ollier's dyschondroplasia is developmental in nature. Chondromatous lesions are encountered in several bones. The age range is between 1 to $12 \mathrm{yrs}$. These lesions are generally distributed on one side of the body. These may lead to shortening of the limb. Radiological findings include cortically oriented lesions with calcifications. The hook sign indicates that it is cartilaginous lesion. This lesion prevents normal growth of the bone and may lead to shortening of the bone (Fig. 24ab).

Chondroblastoma is a benign cartilage tumor and constitutes $1-3 \%$ of all bone tumors. Male and Female ratio is 2:1 and is encountered in 2 nd \& 3rd decades. Long \& short tubular bones are frequently affected. Radiologically a lytic area is noted in the epiphysis or metaphysis, or the lesion may involve both epiphysis and metaphysis. Chondroid calcification is present in $1 / 3^{\text {rd }}$ of cases (Fig. 25ab). When it is primarily located in the metaphysis periosteal reaction may be noted (Fig. 26). In about $1 / 3^{\text {rd }}$ of the cases aneurysmal bone cyst may be associated, MRI helps in demonstrating fluid - fluid levels (Fig. 27ab).

Chondromyxoid fibroma is a rare benign cartilaginous tumor although it is also included in fibrogenic tumors. It is encountered in $2^{\text {nd }}$ and $3^{\text {rd }}$ decades. It occurs in the metaphysis of long tubular bones as well as short bones of the feet. Radiologically, it is a lytic expanding lesion tending to be eccentric. Trabeculations may 

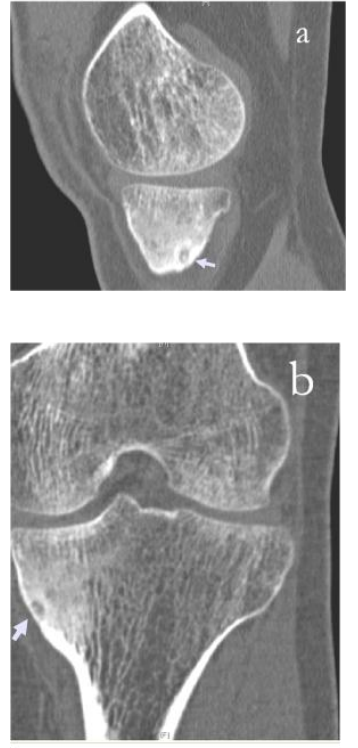

Fig. 9ab: CT showing a tiny osteoid osteoma which is missed on radiographs.

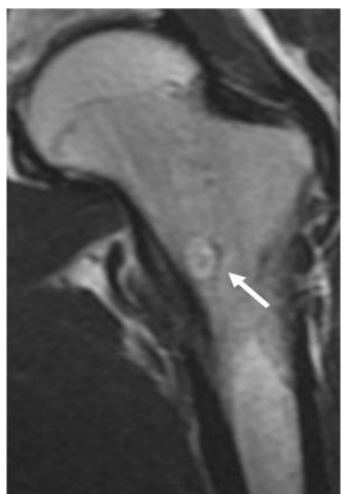

Fig. 10: MRI femur showing the nidus in osteoid osteoma.

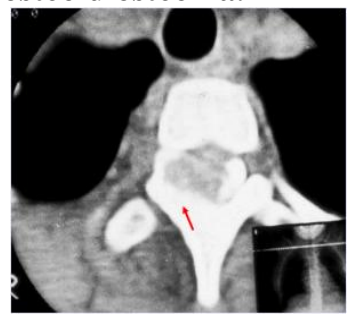

Fig.11:Osteoblasto-ma with large lytic area in the lamina of thoracic vertebra with irregular osteoid matrix.

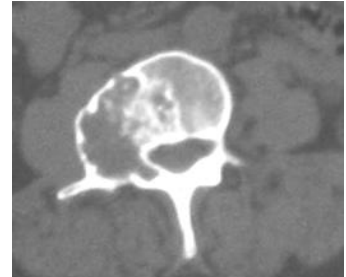

Fig.12: CT osteoblastoma lumbar spine. Note the large lytic area with new bone formation.

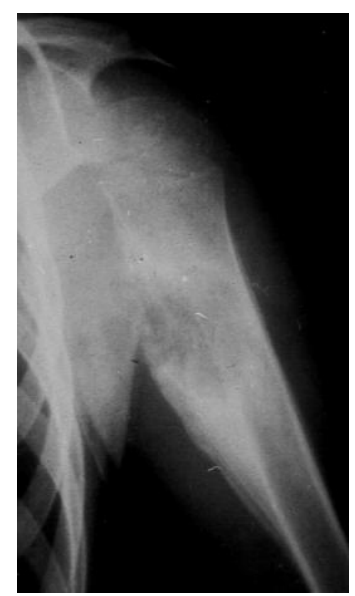

Fig.13: Osteo-

blastoma with a large lytic area with periosteal reaction in the proximal end of humerus.

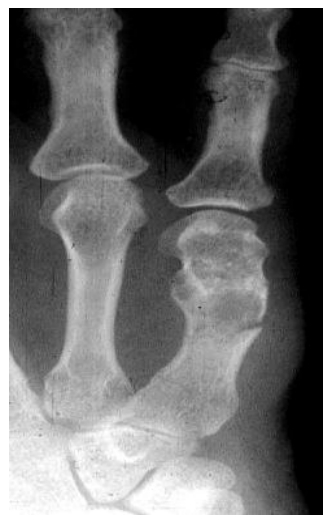

Fig.14: Enchondroma of fifth metacarpal with pathological fracture.

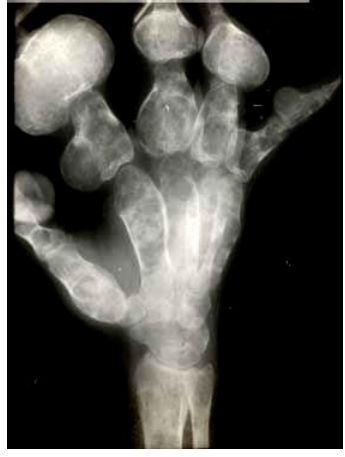

Fig. 15: Multiple enchondromata in the hand.

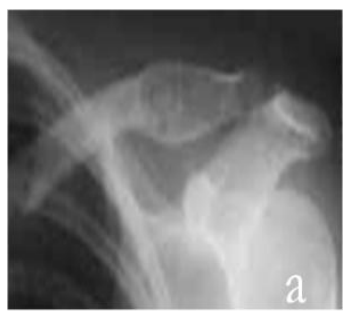

Fig 16a: Enchondroma in Clavicle.

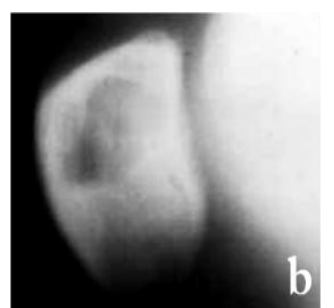

Fig 16b: Enchondroma in patella.

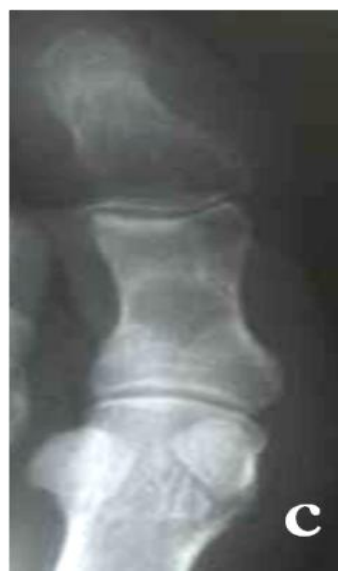

Fig 16c: Enchondroma in big toe.
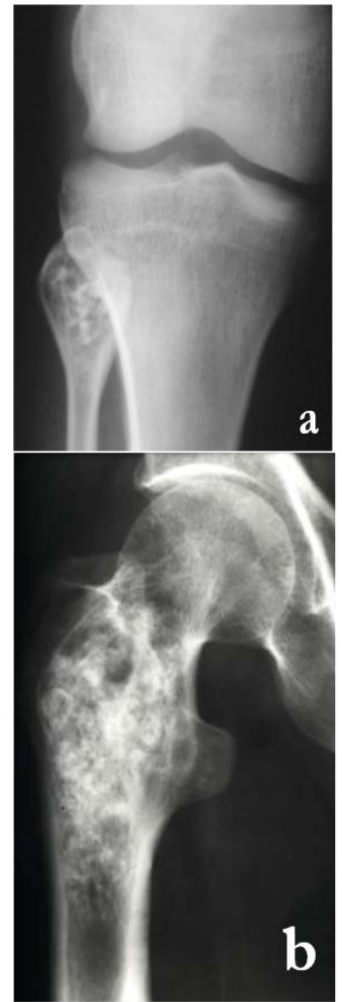

Fig.17ab: Calcified enchondromata, a. head of fibula,

b. intertrochanteric area of femur.

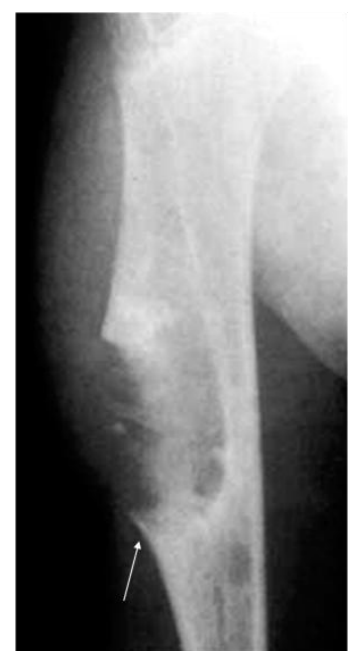

Fig.18: Intracortical chondroma showing calcifications and the presence of hook inferiorly. 


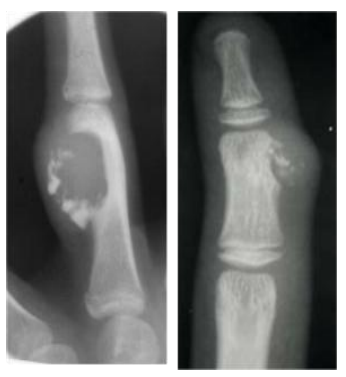

Fig. 19: Peristeal chondroma in the phalanges.
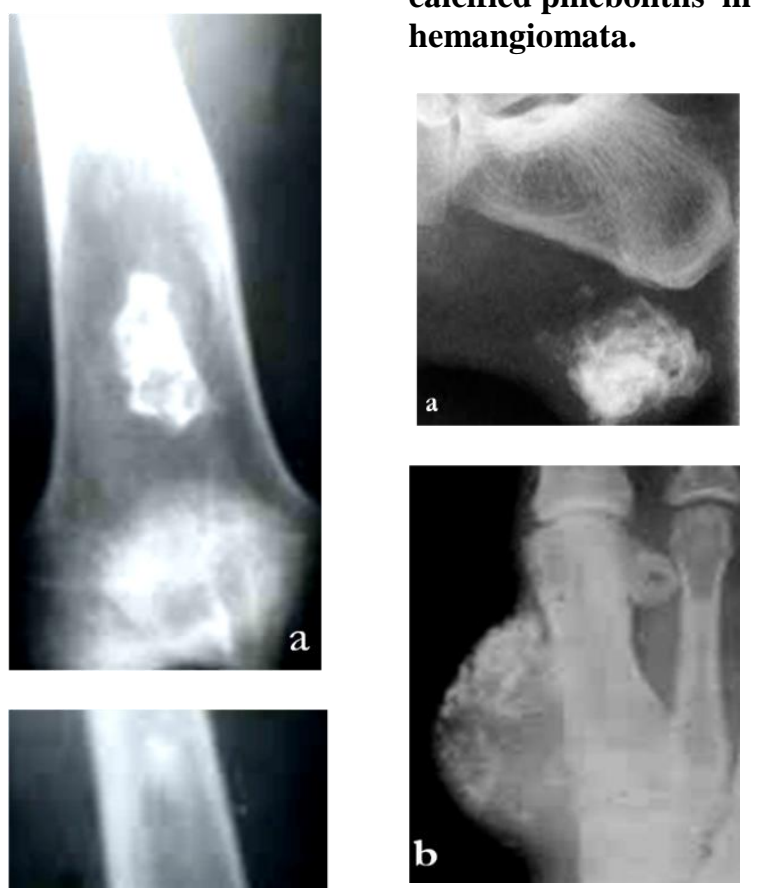

Fig. 22ab: a. heel, b. hand - Soft tissue enchondromata.

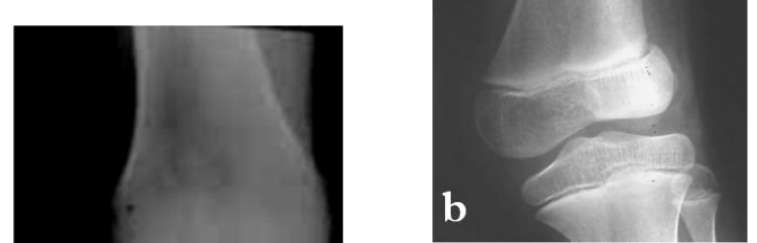

Fig.24ab: Ollier's dyschondroplasia - a. hand, b. femur. Note the hook sign as well as the enchondromata.

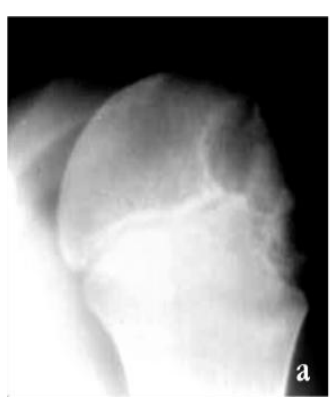

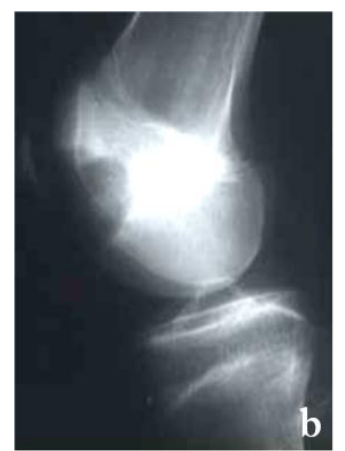

Fig. 25ab:

Chondroblastoma in a. humeral head, b. lower end of femur.

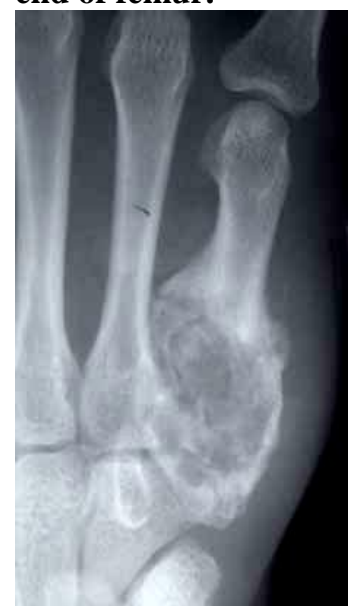

Fig. 26:

chondroblastoma with periosteal reaction at the base of the fifth metacarpal. Note the pathological fracture.
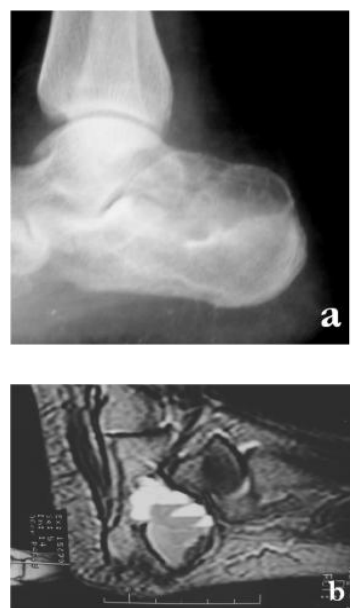

Fig. 27ab:

Chondroblastoma of calcaneum. a. plain film, b. MRI showing fluid fluid levels indicating secondary ABC. 
be seen with endosteal sclerosis (Fig. 28). This is one of the benign aggressive lesions simulating radiologically an aneurysmal bone cyst. Calcification is rarely noted, although punctate calcifications are noted histologically. Advanced imaging does not really help in the diagnosis.

Solitary osteochondroma (exostosis) is generally encountered in the first four decades. When a single lesion is noted it is appropriate that the whole body is investigated for detecting multiple lesions. Radiologically, it occurs in the metaphysis and diametaphysis and projects out of the underlying the bone. It may be sessile or pedenculated. The femora and tibia are common sites. The medularic cavity is in continuity of the parental bone. In long bones these lesions grow away from metaphysis. Growth takes places in the cartilaginous cap. Growth, generally diminishes after skeletal maturity (Fig. 29abc).

Multiple osteochondromata (exostoses) are hereditary and autosomal dominant. These are encountered in younger age groups. They are bilateral and often symmetrical. Radiologically, the findings are that of solitary exostosis. However, since they are developmental, shortening and deformity of limbs may be noted (Fig. 30abcd).

Complications include fracture, bursal formation, compression of nerves and vessels and malignant transformation (Fig. 31ab). While in solitary exostosis / Enchondroma the incidence of malignancy is $1 \%$ (Fig. 32) in multiple exostoses the incidence may go upto $10-15 \%$. In Maffucci syndrome the incidence is $20-30 \%$ which includes non osseous tumors.

Post traumatic exostosis has been described and when it takes the shape of a tent it is named as Turret exostosis. Another exostosis is due to osteoperiostitis or Nora's lesion as a sequel to trauma (Fig. 33ab). Post radiation osteochondromata have also been described.
Trevor's Disease (Dysplasia epiphysialis hemimelica) is a disorder with eccentric growth of ossification centre associated with calcified mass. It may be considered as epiphyseal osteochondroma. This may be hereditary. Deformity of joint may develop. Generally, monoarticular but more than one joint may be affected. Talus and distal femoral epiphyses are most commonly involved. Radiologically, focal calcifications occur at the epiphysis or apophysis. Affected centres may become hypertrophied resulting in severe deformities (Fig. 34abc).

Synovial chondromatosis may be primary or secondary. Primary synovial chondromatosis may be intra articular or extraarticular (Fig. 35). Secondary synovial chondromatosis is due to advanced degenerative arthritis. The degenerated and fragmented cartilage and synovium may be separated and form a nidus for secondary synovial osteochondromatosis. Primary synovial chondromatosis is due to metaplasia of the synovium which gets fragmented and nourished by synovial fluid and eventually calcifies and ossifies and form chondromatosis.

Fibrous tumors of the bone are listed in table VI

Table VI: Fibrous tumors of the bone.

- Fibrocortical defect

- Nonossifying fibroma

- Ossifying fibroma

- Desmoplastic fibroma

- Osteofibrous dysplasia

- Fibromatosis

- Neurofibroma

- Fibrous dysplasia

- Chondromyxoid fibroma

Benign Fibrous tumors may include fibrocortical defect, nonossifying fibroma, ossifying fibroma and fibroxanthoma. Fibrocortical defects are developmental in nature and not really tumors. These are common between 4 and 10 years and 

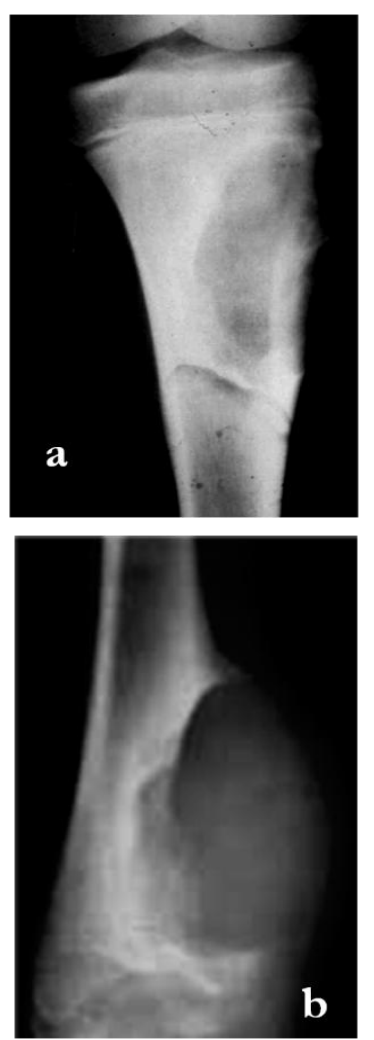

Fig. 28ab:

Chondromyxoid

fibroma of a. proximal tibia, b. distal tibia

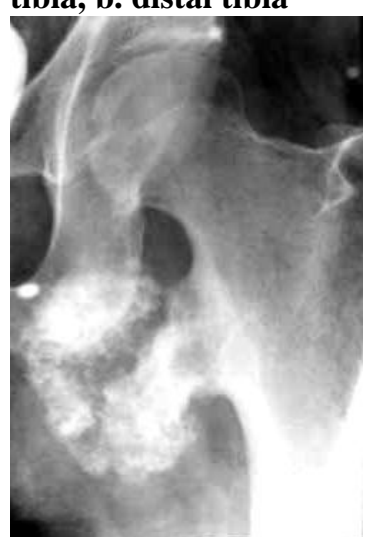

Fig. 29a: Exostosis of lesser trochanter of femur

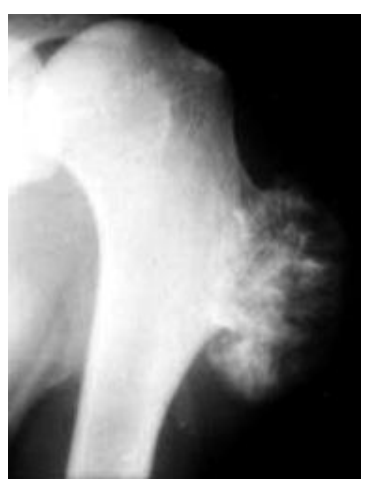

Fig. 29b: humerus. Note cauliflower like osteochondroma

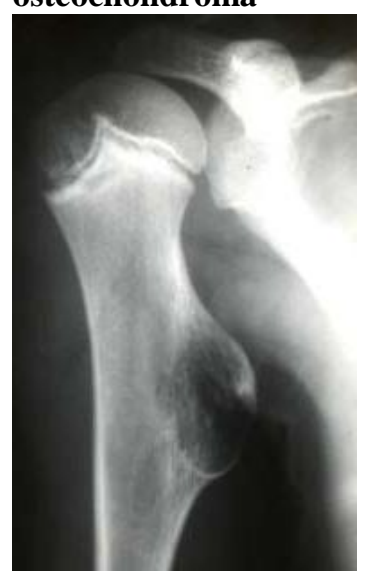

Fig. 29c: Sessile Exostosis of humerus seen end on

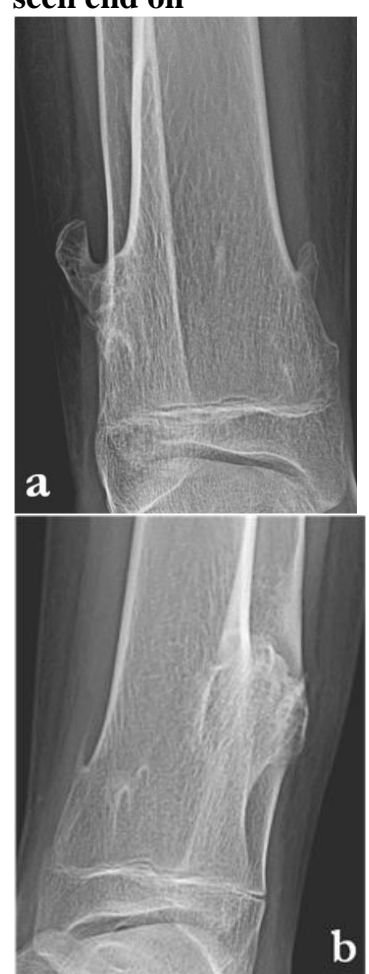

Fig. 30ab: Bilateral osteochondromata. Note hook like projections

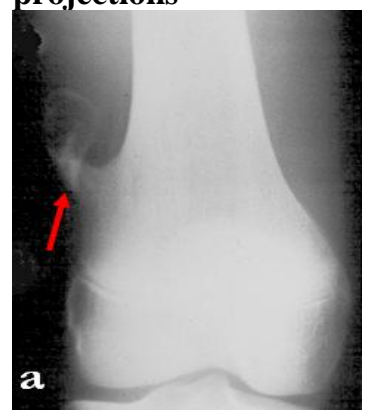

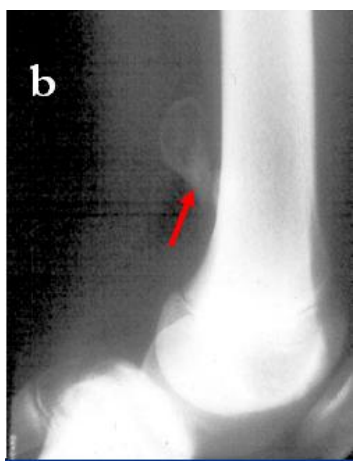

Fig. 31ab: Fracture of the pedinculated exostosis

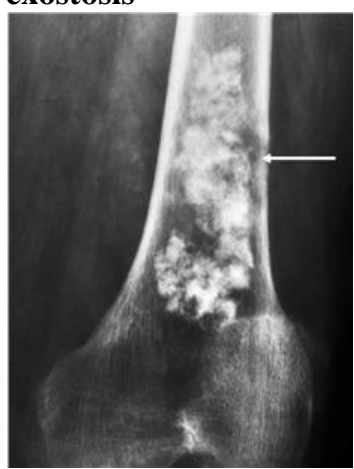

Fig. 32: Benign enchondroma undergoing malignancy in the lateral aspect eroding the cortex.

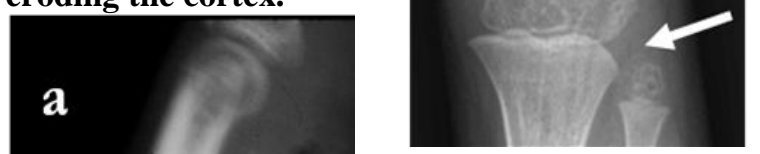

Fig. 34abc: Trevor's disease involving a. Femoral head, b. Talus and c. Knee

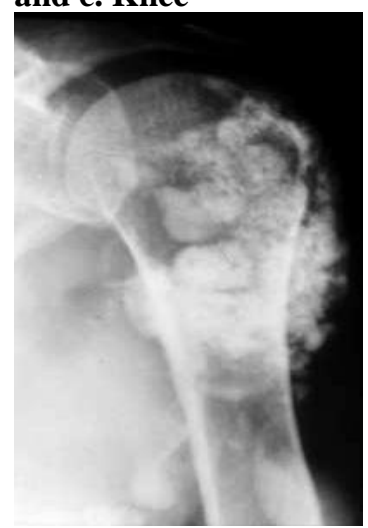

Fig. 35: Primary

synovial

chondromatosis of the shoulder.
Fig. 33ab: a. Nora's lesion due to osteoperiostitis, b. Turret exostosis due to previous trauma 
predominantly present in femur and tibia. Radiologically, they appear as elliptical lucent defects with sclerotic rim (Fig. 36ab). As the child grows these disappear spontaneously.

Nonossifying fibroma is the most common benign fibrous lesion seen in $2^{\text {nd }}$ and $3 \mathrm{rd}$ decades. It is classically seen at the metaphysis of long bones, generally in lower limbs. A pathological fracture generally brings the patient to medical attention.

Radiologically, a cortically oriented eccentric radiolucent defect with a narrow zone of transition is noted. The margins are lobulated and sclerotic with multiple bone ridges. More aggressive lesions persist to adult life and migrate to diaphysis. This is one lesion that the radiologist should be firm in diagnosis and should not advise the surgeon to resect (Fig. 37ab), CT and MRI are unnecessary.

Ossifying fibroma is encountered in the first two decades and mainly involves tibia, although fibula may also be involved. The child complains of bowing of tibia. Radiologically, a well defined lesion with mixed, lytic and sclerotic areas (Fig. 38ab), affecting the middle third of the tibia and produces anterior bowing. Rarely peasudoarthrosis may develop. Spontaneous resolution may occur. Surgical intervention often results in recurrence. When aggressive it may simulate osteofibrous dysplasia (Fig. 39).

Desmoplastic fibroma occurs in $2^{\text {nd }}$ and $3^{\text {rd }}$ decades. Long bones are mostly affected. However innominate bone, scapula, mandible and rarely spine may be involved. Radiologically, it is an aggressive lytic lesion with endosteal erosion and cortical expansion. It may occur in the metaphysis and diametaphysis. The zone of transition may be wide, it is difficult to make a firm diagnosis on plain films alone (Fig. 40).

Cortical desmoid is a tumor like lesion resulting from avulsive injury of the adductor magnus musculofibrous segment. It is located on the posteromedial surface of distal end of femur (Fig. 41ab). Irregular new bone formation simulates osteogenic sarcoma. Biopsy also may be mistakenly reported as malignant bone tumor.

Benign fibrohistocytoma is rare and encountered in $3^{\text {rd }}$ and $4^{\text {th }}$ decades. Radiologically, a lytic well defined lesion is noted with narrow zone of transition. Sclerotic border may be seen and the lesion is eccentrically located. It is believed to be a regressed giant cell tumor.

Fibroxanthoma is a histological diagnosis but radiologically resembles fibrous dysplasia with a lytic area and dense rind (Fig. 42ab)

Infantile Fibromatosis is seen in early childhood. Radiologically, soft tissue swellings are noted with metaphyseal defects in long bones. Minimal periosteal reaction may also be noted (Fig. 43ab).

Chondromyxoid fibroma has been dealt in cartilaginous lesions.Fibrous dysplasia belongs to the category of skeletal dysplasia. Hence it is not described here.

Vascular tumors of bone are classified in table VII. The most common lesion is hemangioma followed by hemangiopericytoma.

Table VII: Vascular tumors of bone.

- Cavernous

- Capillary

- Venous

- Glomus tumor

- Hemangiopericytoma

Hemangioma of the bone is encountered in $4^{\text {th }}$ and $5^{\text {th }}$ decades. The vertebra is more common but skull, long bones, flat bones and soft tissues may be involved. It comprises about $1 \%$ of skeletal neoplasms. Radiological findings depend upon the site of 


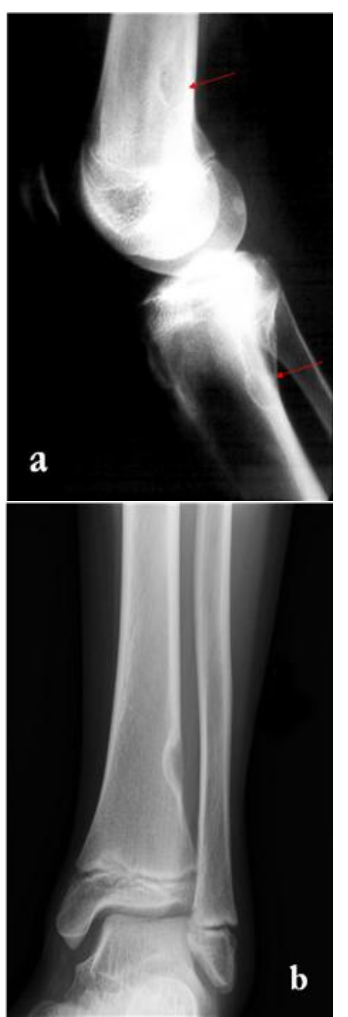

Fig 36ab. Fibro Cortical defects. a. femur and tibia and b. tibia

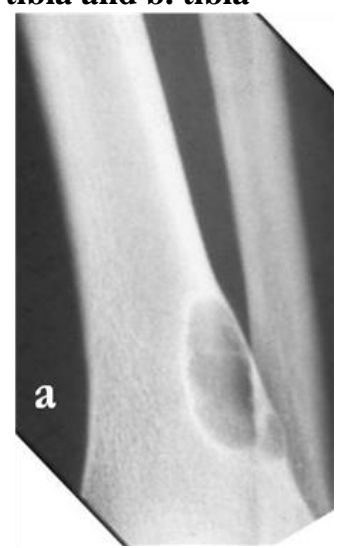

Fig. 37a: Nonossifying fibroma in tibia.

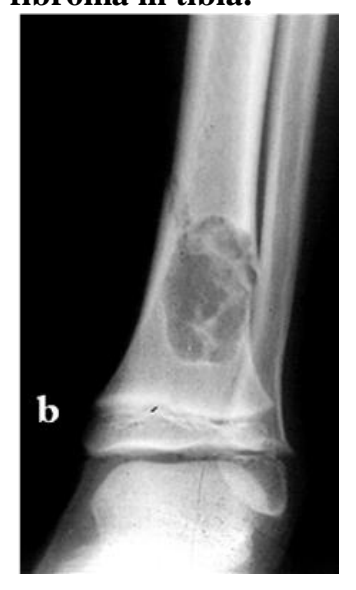

Fig. 37b:. Nonossifying fibroma in tibia with a fracture

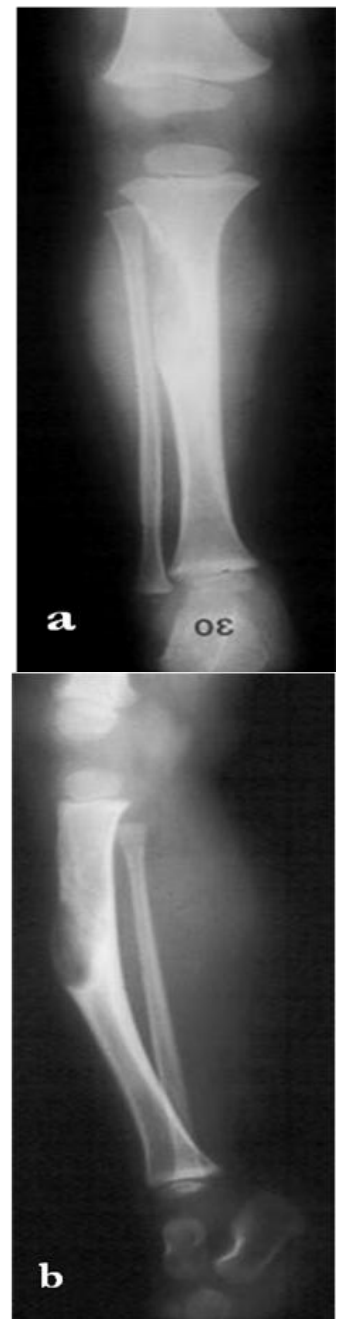

Fig. 38ab: Ossifying fibroma involving cortex of middle third of tibia

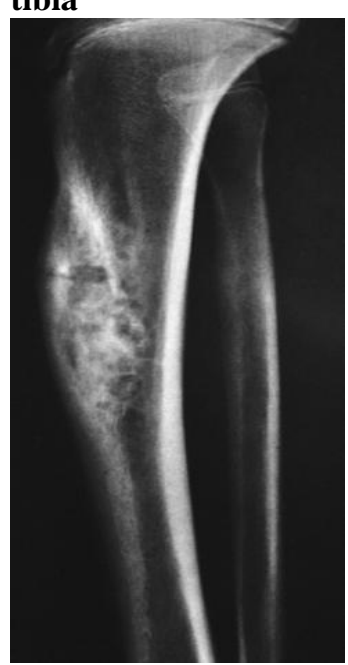

Fig. 39: Osteofibrous dysplasia with lytic and sclerotic changes

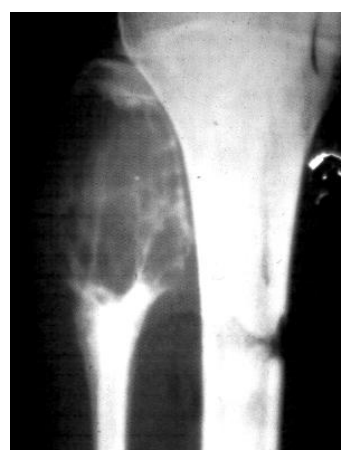

Fig.40: Desmoplastic fibroma of fibula difficult to differentiate from $A B C$

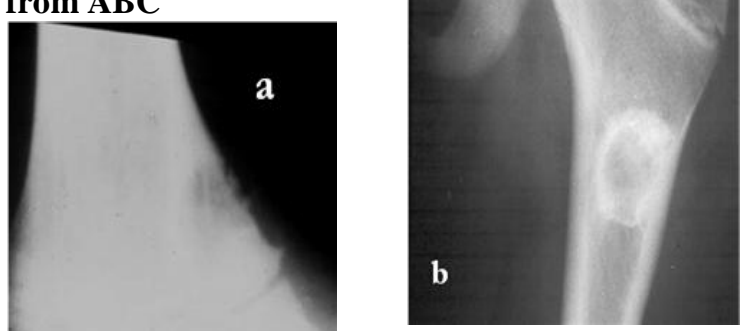

Fig. 42ab:

Fibroxanthoma of

femur

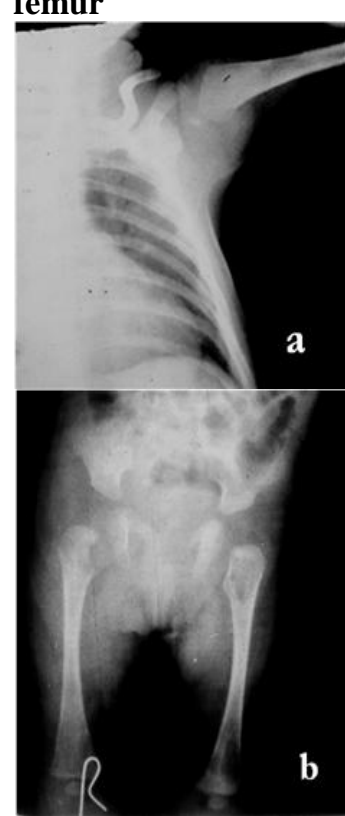

Fig. 41ab: Cortical desmoid in the lower end of the femur on medial aspect due to avulsion injury of few fibres of adductor magnus.
Fig. 43ab: Infantile Fibromatosis involving shoulder and proximal ends of femora

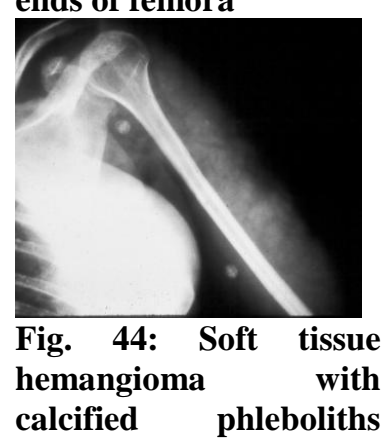



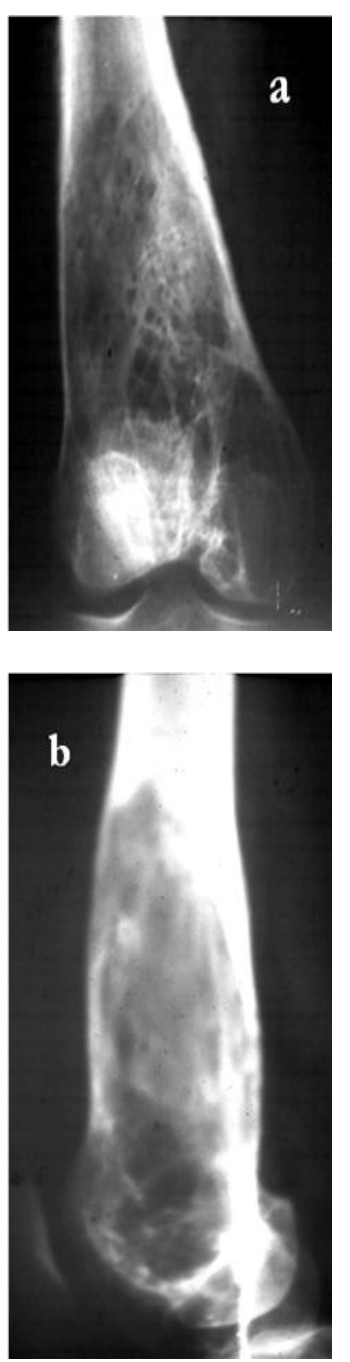

Fig. 45ab: Hemangioma of lower end of femur
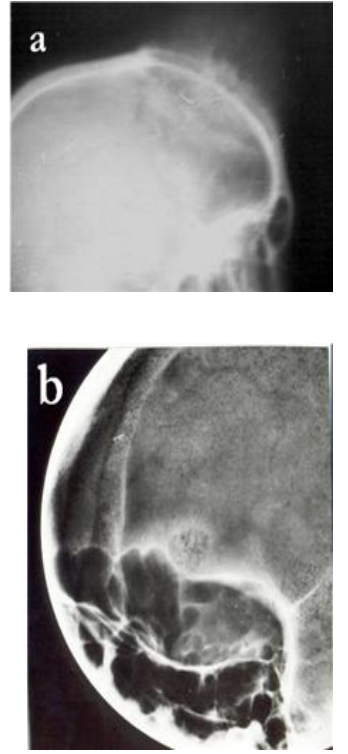

Fig. 46ab: Hemangioma of skull. a. "Sun Burst" appearance, b. "spoke wheel"

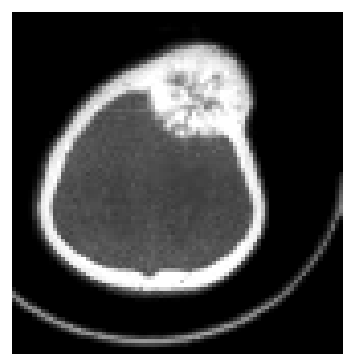

Fig. 46c: Hemangioma CT of skull
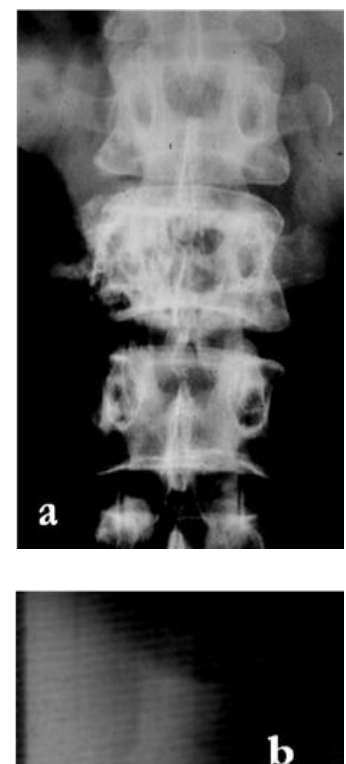

b

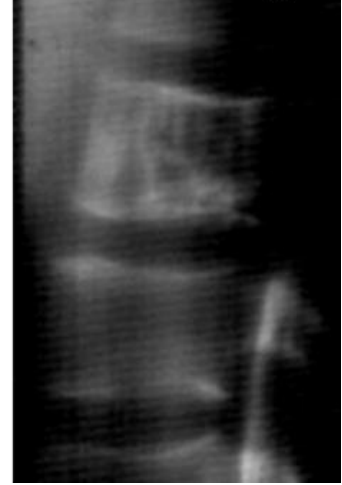

Fig. 47ab: Vertebral Hemangioma. a. plain, b. tomogram

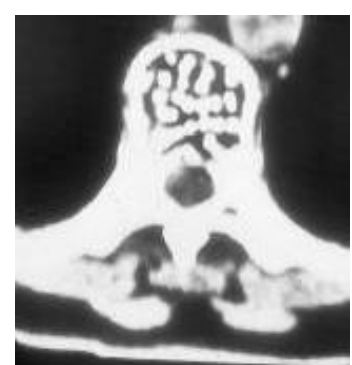

Fig. 48: CT -

Hemangioma of vertebra. CT shows polka dot appearance

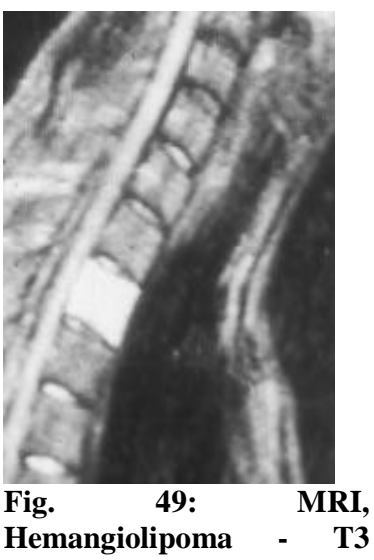

Hemangiolipoma bright signal

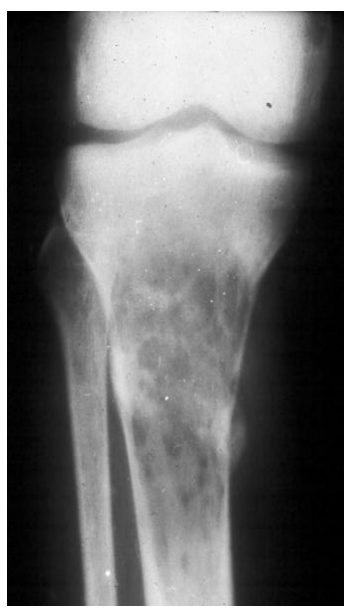

Fig.50:

Hemangiopericytoma involving the tibia

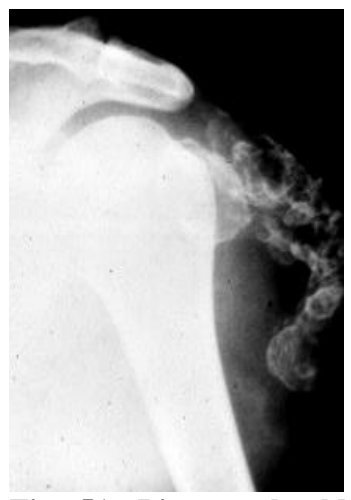

Fig. 51: Lipoma shoulder with soft tissue calcification

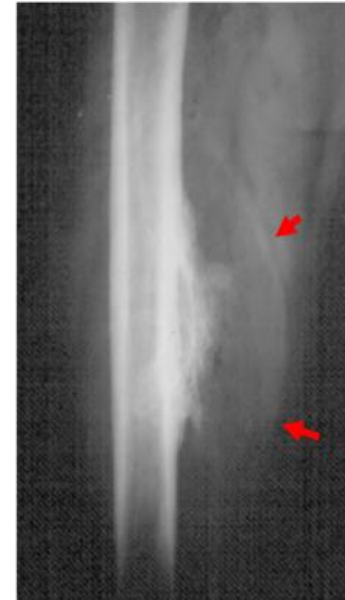

Fig. 52a: Paraosteal lipoma of femur

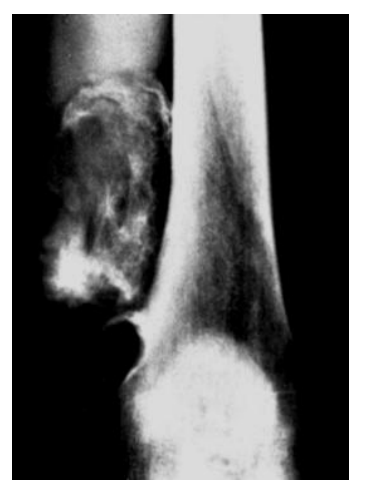

Fig. 52b: Paraosteal lipoma of femur

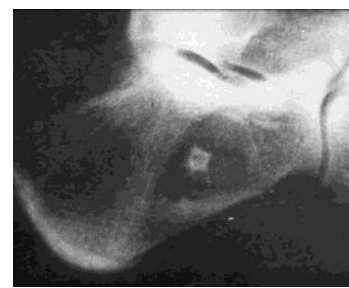

Fig. 53: Lipoma of calcaneum with central calcification 
hemangioma. Soft tissue hemangioma may show calcified phleboliths (Fig. 44).

Hemangioma of a long bone shows honeycombing with vascular channels. However, the radiological findings are not classical (Fig. 45ab).

Hemangioma of skull is often seen in frontal and parietal bones. Radiological appearances have been described under "spoke wheel", sun burst or honeycombing patterns. Large vascular channels adjacent to the lesion may be seen (Fig. 46abc).

Vertebral hemangioma is more common particularly in elderly women. Radiologically, coarse vertical striations simulating corduroy or jail bar appearance best seen in the lateral view (Fig. 47ab). CT appearance is characteristic and is called Polka dot appearance (Fig. 48). In MRI scanning bright signal is noted due to the presence of fat (Fig. 49). Vertebral hemangiomas are also called hemangiolipomas. Most of these are incidental findings.

Cystic angiomatosis is a rare entity involving several bones with multiple hemangiomata. Sclerosing hemangiomatosis is also rare with major component being sclerosis due to reactive new bone.

Hemangiopericytoma is another vascular lesion which produces mainly lytic areas with presence of varied trabeculae. It may expand the bone. It is difficult to diagnose on plain films alone (Fig. 50)

Miscellaneous lesions such as lipoma of soft tissue produces a lucent area in the plain films. It is well demarcated. Calcification may rarely be seen (Fig. 51).

Paraosteal lipoma is a rare tumor occurring in $4^{\text {th }}$ and $5^{\text {th }}$ decades. Radiologically, a lucent superficial soft tissue lesion adjacent to cortex is noted. Calcification and ossification are common and periosteal proliferation may be seen at the attachment to the shaft (Fig. $52 \mathrm{ab})$.

Intra osseous lipoma is also rare and may occur in any bone. However, when it occurs in the calcaneum radiological appearance is classical. A lucent lesion is noted in the body of the calcaneum with central calcification or ossification (Fig. 53).

Ganglionic cyst is often seen in adults in the subarticular area of joint. Radiologically, a lucent lesion with sclerotic rim is noted (Fig.54ab).

\section{Further Reading:}

1. Rockwell M.A. et al. Periosteal Chondroma J. Bone Jt. Surg. (Am.) 1972;54(4):102-108.

2. Murray Ronald O., Jacobson Harold $\mathrm{G}$ and Stoker Dennis $\mathrm{J}$ et al. The Radiology of skeletal disorders. $3^{\text {rd }}$ edition, Churchill Livingstone, New York, 1990.

3. David C. and Dahlin et al. Bone tumors. $3^{\text {rd }}$ edition, Charles $C$ Thomas, U.S.A., 1978.

4. Greenfield GB: Radiology of Bone Disease. Philadelphia, JB Lippincott, 1975.

5. Spjut Harlan J., Dorfman Howard D., Fechner Robert E. And Ackerman Lauren V et al. 2nd series; Tumors of bone and cartilage, New York, 1971.

6. Resinick and Niwayama et al. Diagnosis of bone and joint disorders. 3 vol: Philadelphia, 1982.

7. Subbarao Kakarla and Satish K. Bhargava et al. Diagnostic Radiology and Imaging, revised edition under print, $2012-13$. 